\title{
A new 2D Hénon-logistic map for producing hyperchaotic behavior
}

\begin{abstract}
Derived from the two-dimensional (2D) Hénon map and the one-dimensional (1D) Logistic map, this paper proposes a new 2D hyperchaotic map, called the 2D Hénon-Logistic map (2D-HLM). The dynamics of the 2D-HLM are investigated by means of equilibria, stability analysis, trajectory, Lyapunov exponent, and bifurcation diagram. Mathematical analysis reveals that the 2D-HLM has four unstable equilibria. Besides that, it has wide chaotic and hyperchaotic behaviors with very limited periodic windows. To evaluate the complexity performance of the 2D-HLM, Approximate entropy is used to analyze its time series. Consequently, the 2D-HLM exhibits extremely complex nonlinear behavior. With all of these attributes, the 2D-HLM would be very appropriate to produce a pseudo-random number generator that can be used in chaos-based cryptographic applications.
\end{abstract}

Keyword: Equilibria; Stability analysis; Lyapunov exponents; Hyperchaotic behavior; Approximate entropy 